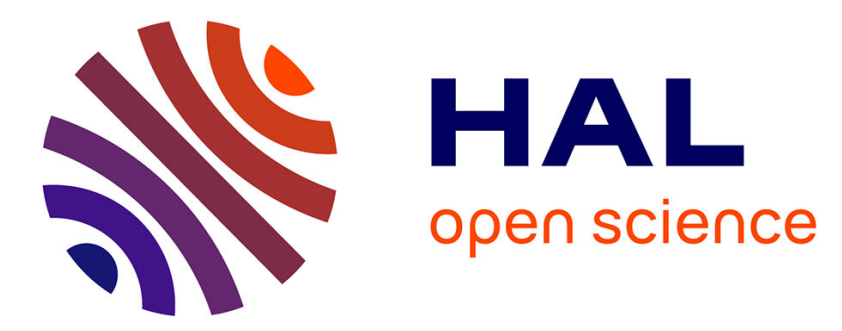

\title{
Caractérisation mécanique des anches simples d'instruments à vent
}

\author{
E. Marandas, V. Gibiat, Ch. Besnainou, Noël Grand
}

\section{To cite this version:}

E. Marandas, V. Gibiat, Ch. Besnainou, Noël Grand. Caractérisation mécanique des anches simples d'instruments à vent. Journal de Physique IV Proceedings, 1994, 04 (C5), pp.C5-633-C5-636. 10.1051/jp4:19945136 . jpa-00252813

\section{HAL Id: jpa-00252813 https://hal.science/jpa-00252813}

Submitted on 1 Jan 1994

HAL is a multi-disciplinary open access archive for the deposit and dissemination of scientific research documents, whether they are published or not. The documents may come from teaching and research institutions in France or abroad, or from public or private research centers.
L'archive ouverte pluridisciplinaire HAL, est destinée au dépôt et à la diffusion de documents scientifiques de niveau recherche, publiés ou non, émanant des établissements d'enseignement et de recherche français ou étrangers, des laboratoires publics ou privés. 


\title{
Caractérisation mécanique des anches simples d'instruments à vent
}

\author{
E. MARANDAS, V. GIBIAT, Ch. BESNAINOU* et N. GRAND
}

Laboratoire Ondes et Acoustique, Université Paris 7, ESPCI, URA 1503 du CNRS, 10 rue Vauquelin, 75005 Paris, France

* Laboratoire d'Acoustique Musicale, UMR, Université Paris 6, CNRS et Ministère de la Culture 9945 T66, 2 place Jussieu, 75005 Paris, France

\begin{abstract}
When modelling woodwinds, when one try to built synthetic reeds one need a good mechanical description of the cane reed. We show that the basic approach in terms of a simple mechanical oscillator is not valid. The correct description must include a viscoplastic characteristic for a wet reed and a viscoelastic one for the dry reed. So it is not possible with classic methods to determine the elastic modulus of a cane reed, and the physical models which include the reed behaviour with a modal description should have to be modify according the equations we present here.
\end{abstract}

\section{Introduction}

Le roseau dans lequel sont taillées les anches d'instruments à vent est un matériau anisotrope et mécaniquement très variable selon sa provenance et les traitements qu'il a subi. Les anches fabriquées dans ce matériau sont par essence des objets uniques dont les propriétés ne possèdent pas la reproductibilité nécessaire à la fois au musicien et au scientifique. Néanmoins à ce jour il n'existe pas de matériaux permettant de fabriquer, au coût dérisoire du roseau, des anches qui donnent satisfaction au musicien. Les défauts courants de l'anche en roseau, sensibilité à l'hygrométrie, à la température, fragilité etc. sont encore largement compensés par les qualités musicales de certaines d'entre elles. De nombreux matériaux ont été utilisés pour tenter de remplacer le roseau: du verre au magnésium de nombreux brevets ont été déposés. Il existe des anches en matériaux de synthèse mais leur utilisations reste marginale.

Nous avons voulu à la suite de nombre d'acousticiens et de musiciens chercher à fabriquer une anche de qualité honorable en matière synthétique. Nous nous sommes appuyés sur la démarche et les prototypes de Backus[1], démarche expérimentale que l'on retrouve chez tous ceux qui ont étudié l'objet acoustomécanique "anche simple en roseau " et qui repose également sur le souci d'intégrer aux modèles de fonctionnement des instruments à vent des caractéristiques physiques liées à l'anche qui soient les plus proches possibles de la réalité. Après avoir supposé que l'anche réagissait instantanément aux variations de pressions acoustique, l'hypothèse la plus physique consiste à la doter d'une élasticité puis d'une masse voire d'un amortissement.

On peut ensuite complexifier le modèle à l'infini et considérer une description modale qui fasse intervenir autant de modes qu'il est possible d'en imaginer. C'est ce travail qu'on réalisé Bouasse [2], Backus [1], Nederveen [3] et plus récemment Casadonte [4].

On pourrait supposer que l'on possède désormais tous les éléments pour synthétiser une anche de qualité. Or même les derniers prototypes de Backus de l'avis de musiciens ne sonnent pas comme du roseau. 
Après avoir constaté la très grande dispersion des résultats concernant le module d'Young et dans une moindre mesure la densité du roseau, nous avons décidé d'effectuer deux types de mesures: élasticité d'une anche montée le plus près possible de ses conditions de fonctionnement normal, et fréquences de résonances. En effet celle-ci sont rarement mesurées dans les mêmes conditions ce qui rend la comparaison délicate. Nous avons donc conçus les dispositifs détaillés ci après en considérant que ces paramètres étaient essentiels pour comparer une réalisation éventuelle à une anche réelle.

\section{Nos mesures sur l'anche simple élastique.}

La plupart des études sur l'anche partent de l'hypothèse suivante: le roseau étant très sensible à l'hygrométrie, il convient de l'étudier sec pour pouvoir le modéliser. Or Nederveen note que la compliance d'une anche mouillée est deux fois plus faible que celle d'une anche sèche, et tous les musiciens évitent de jouer une anche qui n'ait été mouillée au préalable. Nous avons adopté la démarche inverse et nous étudierons le plus souvent une anche en conditions de jeu musical c'est à dire saturée en humidité. Le roseau est un matériau multicouches dont la densité, au contraire du bois, varie de l'extérieur vers l'intérieur; du plus dense et rigide au plus léger et plus souple. Aux petites oscillations c'est la partie légère et souple qui est solicitée et aux grands déplacements ce sont les couches de plus en plus extérieures qui interviennent.

Pour caractériser l'anche il est clair que le seul module d'Young ne suffira pas; le matériau n'est pas homogène transversalement et cette grandeur variera donc avec l'amplitude de déplacement imposé. Nous avons étudié cette variation d'élasticité avec le dispositif suivant. Une balance montée sur un système à micro déplacement permet de mesurer la réaction d'un matériau à un déplacement connu.

Les résultats pour une feuille de composite unidirectionnel carbone-epoxyde montrent bien un comportement linéaire et atemporel de la réaction en fonction du déplacement. La même mesure effectuée sur une anche en roseau permet de constater que le roseau se comporte très différemment: l'évolution n'est plus linéaire. Néanmoins cette mesure est drastiquement entachée d'erreur et il ne saurait être question de l'utiliser pour déterminer un module d'élasticité qui serait une fonction du déplacement. En effet les résultats de la figure 1 qui sont obtenus à déplacement constant montrent que pour un déplacement faible la force de réaction n'est pas constante et varie pendant une durée importante. Il semble même que pour l'anche mouillée on n'atteigne jamais de point stable. Malheureusement le roseau ayant la mauvaise habitude de sécher on rejoint très vite le comportement de l'anche sèche. Les résultats du carbone n'ont pas été reproduits et ne présentent pas de variation avec le temps. Nous pouvons ainsi grâce à une mesure simple affirmer que le comportement de l'anche aux petits déplacements est viscoélastique pour une anche sèche et viscoplastique pour une anche mouillée. Ceci suffit à expliquer les énormes variations des modules d'Young publiés. En effet l'hystérésis important entrainé par ce comportement ne permet pas de mesure fiable et facile. Une autre conséquence est que l'anche va se comporter très différemment de ce que les modèles élastiques suggèrent. Il peut sembler curieux que cette évidence puisse avoir échapper à tous ceux qui ont étudier l'anche en roseau; en effet tous les musiciens (clarinettistes et saxophonistes ) savent parfaitement comment fermer ou ouvrir un bec de manière durable lorsqu'ils utilisent une anche trop faible ou trop forte. Il suffit d'écarter celle-ci de sa position normale sur le bec dans le sens désiré, l'anche conserve cette déformation. De plus la plupart des matériaux naturels, les roches, les bois sont viscoplastiques ou viscoélastiques selon l'amplitude des déformations imposées.

La caractéristique importante du roseau mouillé est de posséder ce caractère dès les plus petits déplacements. Un autre point important vient du lien avec l'humidité. L'anche simple est jouée mouillée, le roseau est alors viscoplastique. L'anche n'est jamais jouée sèche: elle est alors viscoélastique. Un anche sèche (dans une boite à souffler) revient avec retard à sa position de repos, une anche mouillée n'y revient pas.

Un autre point important que révèlent ces mesures concerne l'ordre de grandeur des déplacements observés. On trouve des valeurs de la réaction mesurée de l'ordre de $50 \mathrm{~g}$. pour des déplacements de l'ordre de $.5 \mathrm{~mm}$. . Ces valeurs correspondent à des pressions statiques dans la bouche du musicien de l'ordre de $5000 \mathrm{~Pa}$ compte tenu d'une surface mobile de l'ordre d'un $\mathrm{cm}^{2}$. Ces valeurs sont 2 à 3 fois supérieures a ce que l'on mesure dans la bouche d'un clarinettiste ou d'un saxophoniste en situation de jeu. Les simulations 
numériques et les situations expérimentales qui produisent des déplacements de cet ordre de grandeur sont manifestement irréalistes d'un point de vue musical. On est donc limité à des déplacements de l'ordre de $.1 \mathrm{~mm}$ pour rester dans le domaine de l'instrument de musique. Bien entendu d'autres types de mouvements physiques non musicaux correspondant à des déplacements et des pressions statiques plus grandes peuvent être envisagés sous l'angle de la physique. Ceci se comprend bien quand on observe l'anche en position de jeu. Le simple effet du poids de l'instrument suffit à imposer une position de repos qui ne correspond pas à celle mesurée hors jeu. La latitude de déplacement de l'anche devient donc beaucoup plus faible que ce que les facteurs appellent l'ouverture du bec.

\section{Fréquences de résonances.}

Le second type de données disponibles dans la littérature sur les anches simples concerne les fréquences de résonances. Là encore il est difficile de se faire une opinion cohérente tant les types de mesures sont différents. De l'anche montée dans un étau et pincée qui sonne le la ou le sib4 [2] à l'analyse modale sophistiquée [4] la plupart des mesures ignorent la réalité d'un système mécanique monté sur un bec posé sur la lèvre inférieure du musicien l'ensemble étant maintenu avec la mâchoire supérieure.

La viscoplasticité de l'anche va désormais être une paramètre important. En effet la très longue hystérésis qui y est liée ne permet pas de faire des mesures successives. L'importance de l'humidité du roseau pose également le problème du séchage. Nous présentons ici les résultats obtenus sur une anche de saxophone montée sur son bec. L'anche est d'abord saturée d'eau puis excitée par une choc bref. Le déplacement est mesuré par un préamplificateur Bet $\mathrm{K}$ qui sert d'électrode arrière d'un condensateur dont l'anche serait l'autre électrode. La transformée de Fourier du signal obtenue donne accès à la fréquence d'oscillation. Celle -ci varie bien avec l'hygrométrie (figure 2). On constate également que l'amplitude du signal varie avec l'hygrométrie mais aussi entre deux frappes successives. Ceci provient du fait que là encore l'anche conserve la mémoire des contraintes qu'elle a subi.

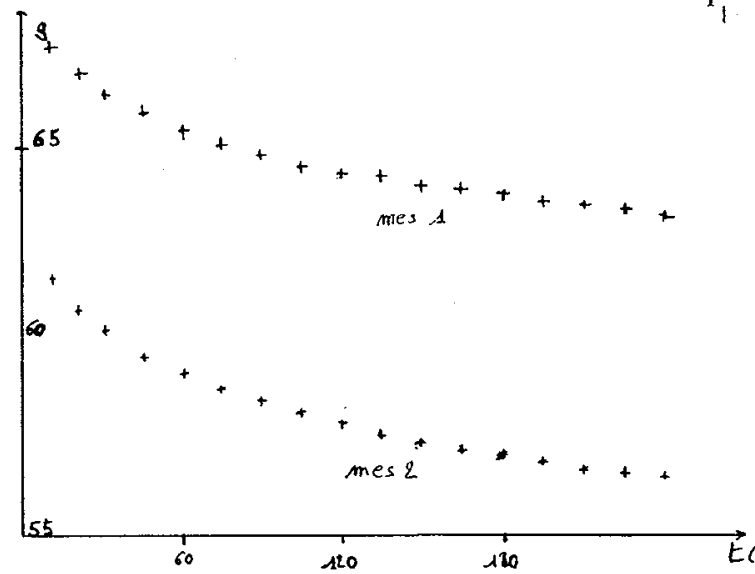

Figure 1

Courbe de relaxation typique de la viscoplasticité mesurée sur une anche de clarinette pour une déformation initiale de $.15 \mathrm{~mm}$.

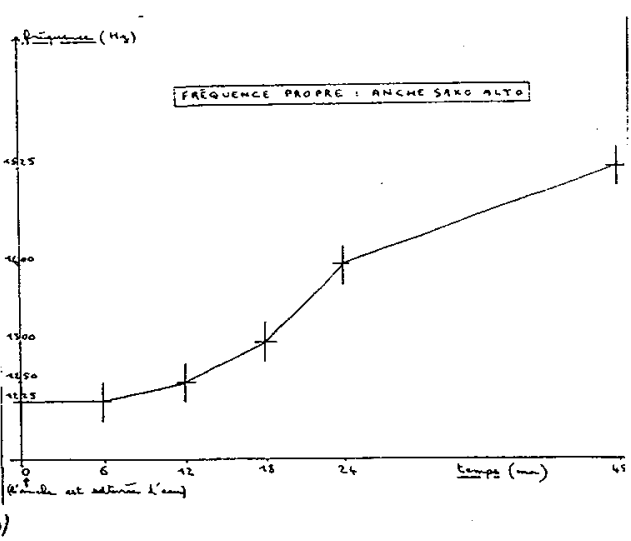

Figure 2

Variation de la fréquence propre d'une anche de saxophone alto en fonction de l'hygrométrie. Les frappes sont effectuées à intervalles de temps éloignés. 


\section{Les équations.}

Il existe des modèles mécaniques simplifiés qui permettent de représenter la viscoélasticité et la viscoplasticité $[5,6]$.

Ces deux modèles permettent d'écrire des équations du modèle de l'instrument de musique différentes de celles classiques:

$$
\begin{aligned}
& p(t)=u(t) * g(t) \\
& u(t)=F_{\text {Nonlin. }}(p(t))
\end{aligned}
$$

En utilisant $y(t)$, pour le déplacement de l'anche le système s'écrit alors en négligeant la masse:

$$
\begin{aligned}
& p(t)=u(t) * g(t) \\
& Z_{0} u(t)=k \cdot y(t) \cdot(y(t)-1) \\
& \ddot{p}+\left(\frac{1}{\tau_{\infty}}+\frac{k_{0}}{\mu_{2}}\right) \dot{p}+\frac{k_{\infty}}{\mu_{2} \tau_{\infty}}\left(p-P_{d}\right)=-\frac{k_{0}}{S} \ddot{y}-\frac{k_{\infty}}{S \tau_{\infty}} \dot{y}
\end{aligned}
$$

où $S$ est la surface de l'anche en mouvement et les paramètres $k$ et $\mu$ correspondent aux paramètres classiques d'un modèle visco plastique simple à deux amortisseurs et deux ressorts $[5,6]$.

Ces équations permettent de faire apparaitre un mode "mou" à fréquence nulle dont le rôle n'est pas élucidé mais pourrait être lié à une brisure de symétrie dans le comportement des équations 3 à 6 .

\section{Conclusion}

Le modèle classique de l'anche se comportant comme un système élastique masse ressort, bien que pertinent pour nombre d'applications, n'est manifestement pas suffisant même en tenant compte des modes les plus variés pour rendre compte de la dynamique de l'anche en roseau. La mise en évidence expérimentale du caractère viscoplastique de l'anche mouillée montre que les déterminations élastiques ne renferment pas toute l'information sur la mécanique de l'anche. L'influence de ce paramètre sur la qualité musicale des anches reste à déterminer, mis on peut déjà noter que les meilleures des anches synthétiques présentent un léger caractère plastique.

\section{Remerciements}

Ce travail a pu être réalisé grâce à une aide du ministère de la culture et nous tenons à remercier Nathalie Piro qui a initié cette étude et Franck Laloë pour de fructueuses discussions.

\section{Références}

[1] J. Backus US patent \#4.337.683 (1982)

[2] H. Bouasse "Instruments a vent tome 2" p73-78 Delagrave Editeur Paris 1930.

[3] C. J. Nederveen "Acoustical aspects of woodwind instruments" p27-30 Frits Knuf Editeur Amsterdam (1967)

[4] D. Casadonte "The perfect clarinet reed? vibrational modes of realistic clarinet reeds" J. Acoust. Soc. Am. 94 (1993) p1807

[5] J.M. Dorlot, J.P. Bailon, J. Masounave "Des matériaux" pp171-173 Editions de l'école polytechnique de Montréal (1986)

[6] T. Bourbiè, O. Coussy, B. Zinszner "Acoustics of porous media" pp99-143 Editions Technip Paris (1987) 